\title{
Vulnérabilités sociales et santé sexuelle : représentations, connaissances et comportements de jeunes à Nouméa
}

\section{Fatou Leïty Mbodj}

\section{OpenEdition}

1 Journals

Édition électronique

URL : http://journals.openedition.org/rfst/478

DOI : $10.4000 /$ rfst. 478

ISSN : 2492-3672

Éditeur

Espaces et SOciétés (UMR 6590)

\section{Référence électronique}

Fatou Leïty Mbodj, « Vulnérabilités sociales et santé sexuelle : représentations, connaissances et comportements de jeunes à Nouméa », Revue francophone sur la santé et les territoires [En ligne], Miscellanées, mis en ligne le 13 mai 2015, consulté le 06 avril 2021. URL : http:// journals.openedition.org/rfst/478; DOI : https://doi.org/10.4000/rfst.478

Ce document a été généré automatiquement le 6 avril 2021.

La Revue francophone sur la santé et les territoires est mise à disposition selon les termes de la Licence Creative Commons Attribution - Pas d'Utilisation Commerciale - Partage dans les Mêmes Conditions 4.0 International. 


\title{
Vulnérabilités sociales et santé sexuelle : représentations, connaissances et comportements de jeunes à Nouméa
}

\author{
Fatou Leïty Mbodj
}

\section{Introduction}

1 La Nouvelle-Calédonie est caractérisée par la jeunesse de sa population. En effet, 50\% de ses habitants ont moins de 30 ans (Rivoilan P. et Broustet D., (2009)). Depuis quelques années sans qu'il y ait réellement d'enquête de prévalence des infections sexuellement transmissibles (IST), de nombreuses études partielles ${ }^{1}$ annonçaient une prévalence élevée des IST, notamment dans la population jeune. A ces données pourraient être rajoutée la fréquence des interruptions volontaires de grossesses dans la tranche d'âge des jeunes de 15 à 24 ans et les entretiens avec les professionnels de santé qui attirent l'attention sur l'importance des prises de risque dans cette catégorie d'âge. Dans ce contexte, une étude transversale de prévalence menée en 2012 par l'agence sanitaire et sociale apporte des précisions sur la prévalence des infections sexuellement transmissibles en Nouvelle-Calédonie. Menée auprès de la population générale âgée de 18 à 49 ans, les résultats de l'enquête indiquent que pour les 3 IST ${ }^{2}$ particulièrement étudiée, la prévalence est plus élevée selon certains facteurs sociodémographiques. En effet, le jeune âge, le fait d'être une femme, l'appartenance à la communauté mélanésienne, un faible niveau d'étude et le célibat constituent des facteurs sociodémographiques associés à un plus fort risque d'avoir ces IST. Ces résultats justifient l'intérêt particulier que l'agence sanitaire et sociale a porté et continue de porter aux jeunes en particulier à ceux en situation de précarité, devenus destinataires prioritaires des messages et actions de prévention. Ainsi dans le cadre de l'amélioration des connaissances et des comportements dans les groupes prioritaires, le comité national de lutte contre le sida et l'agence sanitaire et sociale se sont intéressés 
particulièrement aux facteurs de vulnérabilité de cette population du point de vue de la santé sexuelle. C'est dans ce cadre que nous avons réalisé en 2010, une recherche auprès de quatre populations jugées vulnérables dont les jeunes vivant en squat.

Ce texte issu d'un second travail d'enquête qualitatif plus approfondi et plus spécifique sur la population jeune a été réalisé en début 2014. La recherche se focalise sur une population de jeunes non insérés âgés de 18 à 25 ans vivant en squats dans le GrandNouméa. Le choix d'enquêter auprès de cette population se justifie par plusieurs raisons. Les jeunes sont au cœur des discours politiques et sanitaires et pourtant peu d'études qualitatives se sont penchées sur la question de leur santé en général et sur leur santé sexuelle en particulier notamment dans les milieux défavorisés. Une recherche réalisée en 2010 par l'Institut National de la santé et de la recherche médicale (INSERM, 2011) qui porte sur les jeunes en Nouvelle-Calédonie recoupe en partie les résultats de notre présente étude. Cependant, la particularité de notre travail est qu'il cible particulièrement les jeunes kanak, non insérés, qui vivent en squats dans le Grand-Nouméa.

Dans ce texte, nous souhaitons interroger la façon dont s'articulent les dimensions sociales, spatiales et ethniques dans le cadre de la santé et de la santé sexuelle plus précisément. Plus particulièrement nous tenterons de rendre compte de la façon dont les caractéristiques sociales, notamment la rupture des liens, le faible niveau d'étude affectent les connaissances et les comportements de jeunes en situation de précarité dans un espace subordonné à la ville, le squat. Aussi, nous verrons de quelle façon les relations interethniques prennent forme dans la relation de soin et comment l'approche culturaliste voile et réduit la prise en compte par les professionnels des conditions de vie et de la fragilité sociales des personnes dont ils ont la charge.

Dans cette étude, trois facteurs nous semblent devoir être particulièrement distingués.

5 Le premier facteur est l'exclusion sociale et la rupture des liens sociaux et institutionnels auxquels est confrontée cette population de jeunes qui vivent en périphérie de la ville. De nombreuses études, notamment celles de Pierre Chauvin et de Isabelle Parizot (2005) en zones urbaines sensibles, ont démontré comment la dimension territoriale peut impacter la santé des individus. Les auteurs montrent également que l'expérience de certaines ruptures augmente le risque statistique d'être confronté à d'autres difficultés - des difficultés socio-économiques mais aussi de recours aux soins, des problèmes d'identité et des problèmes de santé ressentie. Les auteurs précisent que d'une manière générale, pour les individus, les difficultés rencontrées aux plans social et sanitaire sont d'autant plus problématiques que les formes traditionnelles de sociabilité et de solidarité s'affaiblissent. Lorsque les liens sociaux se distendent, le risque d'être confronté à un enchaînement d'adversités et de ruptures sociales qui se renforcent mutuellement s'accroît. Comme nous le verrons plus loin dans ce texte, une partie conséquente de la population de jeunes kanak qui vivent en squat est confrontée à ces ruptures qui par la disqualification sociale qu'elles entraînent pourraient compromettre entre autres le recours et l'accès aux soins.

Le second facteur est le niveau de scolarisation qui apparait comme un déterminant essentiel des comportements de santé. En effet, de nombreuses recherches mettent en relation le niveau de formation et le recours aux soins ((Chauvin et Parizot (2005), Anderson et Mortensen (2006), Yaogo et al (2014)). Le système scolaire serait le lieu d'acquisition d'outils permettant aux individus de faire face à leurs besoins en santé. Concernant les jeunes qui ont quitté tôt le système scolaire, questionner leurs 
connaissances dans le domaine de la santé et de la santé sexuelle plus particulièrement pourraient révéler d'éventuelles lacunes ou méconnaissances.

7 En dernier point, nous verrons la façon dont l'approche culturaliste, souvent mobilisée par les professionnels de santé, limite les grilles de lecture du comportement des jeunes et favorise leur enfermement dans des stéréotypes peu propices au véhicule de connaissances et à la prise en compte de déterminants sociaux dans leurs recours et accès aux soins. Les études menées en outre-mer, notamment celles de E. Carde en Guyane (2013), souligne les représentations de «l'Autre » à l'œuvre dans la relation de soin et des pratiques différenciées qui en résultent. Nous rendrons donc compte des discours des professionnels de santé en majorité d'origine européenne, pour certains arrivés de la métropole, et la façon dont, dans ce contexte particulier, la différenciation culturelle et sociale opère dans la relation de soins et interfère dans l'établissement d'une relation compréhensive et de confiance entre jeunes kanak et professionnels de la santé.

\section{La population de l'étude}

\section{Les jeunes non-insérés}

8 L'étude s'intéresse particulièrement aux jeunes kanak non-insérés qui vivent en squat dans le Grand-Nouméa. Le mot kanak désigne le peuple autochtone de la NouvelleCalédonie. En 1853, la France s'empare violemment de l'archipel en confinant dans un premier temps la population indigène dans des territoires délimités à cet effet (les réserves) et dans un second temps en la soumettant au régime de l'indigénat, un ensemble de règles spécifiquement destinés aux kanak, afin d'une part de contrôler leurs déplacements et d'autre part de réprimer leurs «infractions ». Poussés par le sentiment d'avoir été marginalisés dans leur propre pays, les kanak lancent en juin 1975 le mot d'ordre d'indépendance kanak tandis que la tenue, en septembre, du festival culturel Mélanésia 2000 est l'occasion d'une forte affirmation identitaire. Dans un contexte de fortes inégalités, de revendication quant à la souveraineté du pays essentiellement portée par des kanak, de revendication de la reconnaissance et de la valorisation de leur culture, et suite à des confrontations violentes entre l'armée et les indépendantistes, les accords successifs de Matignon et de Nouméa tenteront un rééquilibrage pour une intégration plus importante des kanak dans le fonctionnement économique, politique, social et culturel du pays. Malgré cette politique de rééquilibrage pour la construction d'un destin commun, d'importantes inégalités sociales subsistent entre kanak et non-kanak, notamment dans le domaine de l'éducation, l'accès à l'emploi, au logement, inégalités souvent sources de tensions et de revendications sociales et politiques.

Un rapport récent du Conseil économique et social de la Nouvelle-Calédonie révèle que le chômage des jeunes atteint $22,7 \%$ au second semestre 2012 sur ce territoire, soit plus du double de la moyenne nationale (Prévost, 2012). Selon l'Observatoire des territoires (2011), ce taux est en 2010 presque deux fois plus élevé en moyenne dans les départements d'outre-mer (29,5\%) qu'en France métropolitaine (15,2\%). Le chômage touche particulièrement les jeunes kanak. En effet, une étude menée par Catherine Ris (2014), révèle que malgré la réduction des écarts dans l'accès aux diplômes et à l'emploi entre kanak et non-kanak, les inégalités dans ce domaine restent préoccupantes. Selon 
son étude, en 2009, un jeune non-kanak a 3,4 fois plus de chances qu'un jeune kanak d'être en emploi. Elle précise que l'écart entre les communautés est du même ordre lorsque l'on considère le taux chômage qui est en 2009, de $26 \%$ chez les kanak contre $7 \%$ pour les non-kanak. Au-delà des questions d'inégalités qu'elle soulève et des enjeux autour du système éducatif en Nouvelle-Calédonie, cette étude révèle que l'insertion des jeunes kanak en milieu professionnel est compromise.

10 Les jeunes qui vivent en squat sont particulièrement touchés par la déscolarisation et le chômage. Selon l'étude menée par l'INSERM en 2008 « les jeunes qui habitent en squat ont un niveau de qualification scolaire très inférieur à celui des autres jeunes du Grand-Nouméa. Chez les 22 à 25 ans en squat, seulement $20 \%$ ont un baccalauréat alors que les bacheliers représentent $39 \%$ des jeunes de cette tranche d'âge vivant en cité et $59 \%$ de ceux vivant dans les autres types d'habitations du Grand-Nouméa. Inversement, les jeunes sans aucun diplôme y sont fortement surreprésentés : $33 \%$ des résidents en squat de plus de 21 ans sont sans diplôme (12\% du total des jeunes du Grand-Nouméa dans la même tranche d'âge). Si l'on considère la NouvelleCalédonie dans son ensemble - Grand-Nouméa, Sud rural, Nord et Iles - c'est dans les squats que le niveau de qualification scolaire des jeunes est le plus bas ". Cette sortie précoce du système scolaire voire cet échec scolaire, interroge sur la qualité et le niveau de connaissance dont disposent les jeunes pour faire face à leurs besoins de santé et de recours aux soins, notamment dans le domaine de la santé sexuelle.

11 Le taux d'inactivité et de chômage atteignent en squat des proportions très élevées. $42 \%$ des jeunes vivant en squat sont concernés versus $17 \%$ dans le Grand-Nouméa (INSERM, 2008), indiquant la grande précarité qui touche les jeunes des squats.

Dans le cadre de cette étude afin de rendre compte de cette catégorie de jeunes qui ne sont ni scolarisés, ni diplômés, nous avons empruntés aux services statistiques de renseignement comme l'Institut national de la statistique et des études économiques, la catégorie "jeunes non-insérés». Cette catégorie comporte deux caractéristiques qui renforcent la vulnérabilité et la précarité de ce groupe. En premier lieu, les jeunes noninsérés sont en grande majorité des jeunes sortis du circuit scolaire sans diplôme et avec un faible niveau d'étude. Sans diplôme ni activité ou expérience professionnelle, ils oscillent entre contrats à durée déterminée et recherche d'emploi, voire inactivité. En second lieu, ces jeunes sont prédisposés à l'exclusion sociale et aux ruptures des liens sociaux.

\section{Les professionnels de santé}

13 Dans un contexte de revendication de souveraineté principalement porté par les autochtones kanak, la loi organique n ${ }^{\circ}$ 99-209 du 19 mars 1999 établit la liste des missions de l'Etat pour lesquelles il est compétent, celles pour lesquelles il est associé et celles qui relèvent d'un transfert progressif à la Nouvelle-Calédonie. Ainsi, la justice, le maintien de l'ordre et la sécurité civile, la défense demeurent de la compétence de l'Etat sous la responsabilité du Haut-commissaire qui est le représentant de la République sur le territoire. L'Education, transférée depuis janvier 2012 relève au contraire de la compétence du territoire. Il en est de même pour la santé. Cependant en raison d'un déficit de professionnels de santé locaux, ce sont en grande majorité des médecins occidentaux ${ }^{3}$ venus en général de la métropole qui sont en charge du soin et de la prise en charge médicale de la population locale, dont il faut noter la grande diversité ethnique ${ }^{4}$. 


\section{les lieux de l'étude}

\section{Les squats}

14 Le Grand-Nouméa (Nouméa et sa périphérie) concentre les $2 / 3$ de la population calédonienne et continue d'attirer l'essentiel des migrations (INSEE, 2011). L'ensemble des ethnies y est représenté ${ }^{5}$. Nous avons choisi de travailler dans les squats, convaincus que les dimensions sociales, sanitaires et spatiales des sociétés sont imbriquées.

15 Les zones dénommées "squats» dans le Grand-Nouméa désignent une forme d'habitats spontanés en périphérie de Nouméa, habitats présents depuis une trentaine d'années dans le paysage urbain de la capitale. De nombreuses personnes essentiellement de la communauté kanak quittent la tribu ${ }^{6}$ (souvent située en zone rurale) pour travailler et habiter en ville. La cherté des loyers à Nouméa et les modèles locatifs des cités peu adaptés au mode de vie océanien ${ }^{7}$ incitent la plupart d'entre eux à investir ces quartiers d'habitats spontanés en zones non encore loties. Selon l'enquête Taylor Nelson Sofres (TNS, 2008) 5\% de la population du Grand-Nouméa vivent en squat. Les squats accueillent aujourd'hui plus de 8000 personnes dans une agglomération d'environ 160000 habitants (TNS, 2008). Même si la population des squats n'est pas un ensemble homogène, quelques caractéristiques y sont constantes. L'une des particularités de ces logements précaires est qu'ils abritent en grande majorité la population autochtone kanak alors qu'en métropole, les squats sont occupés en majorité par les populations migrantes notamment celles issues d'Afrique subsaharienne (Quercy, 2002). L'autre particularité réside dans l'activité exercée par ses habitants. Cette activité y serait peu stable, la majeure partie de cette population ayant un niveau de qualification inférieur à celui des autres jeunes du Grand-Nouméa, elle occupe un emploi précaire ou n'a pas d'emploi (INSERM, 2008). Le choix du squat se justifie donc en partie parce que ces lieux permettent d'accéder plus aisément à la population jeune non-insérée qu'on ne peut approcher dans les cadres habituels d'enquêtes (institutions scolaires ou universitaires, lieux de travail..).

Les conditions de vie dans les quartiers marginalisés ont des conséquences directes ou indirectes sur la santé des populations qui y vivent. La présence fréquente des squats dans des zones mal assainies (mangroves, zones marécageuses), l'insalubrité de l'eau du à son mauvais stockage (en raison de coupure d'eau fréquente), le manque d'hygiène globale (notamment dans la gestion des déchets) exposent avec une plus grande fréquence la population de la plupart des squats aux maladies telles que la galle et autres maladies de peau, les problèmes bucco-dentaires, les affections ORL, les diarrhées chez les enfants, l'hépatite virale, la dengue et la leptospirose (Serve, 2009).

17 Pourtant la vie dans ses squats n'est pas toujours à l'image des bidonvilles d'Amérique Latine ou d'Europe. En effet Dussy (2005) souligne que ces derniers se distinguent de ceux de Nouméa par le caractère extrême de la précarité, de la misère, leurs tailles et densités. Le squat à Nouméa n'est pas toujours perçu par ceux qui y vivent comme un lieu hostile. Passa (2010) souligne dans la société kanak, le lien entre l'identité et l'espace. L'espace définit l'identité de la personne. Pour les personnes qui ne sont pas originaires de Nouméa, le challenge est de trouver du sens à leur habitat en ville, sens qui donne aussi un sens à leur identité. Cela nécessite de leur part une formulation et 
une réappropriation autres de l'espace, mais aussi un exercice de redéfinition de leur identité et de leur positionnement au monde. Cette quête de sens peut en partie expliquer leur adoption du mode de vie en squats qui leur permet de reproduire à leur échelle un mode de vie qui leur ressemble, une façon d'investir la ville. Certains de ses occupants y reproduisent certaines fois la vie en tribu avec une réplique de ses règles dans l'organisation interne des squats, un regroupement sur la base du clan comme en tribu et un aménagement d'espaces suffisants et distincts pour les différentes fonctions et activités (loisirs, agriculture, culte). Cependant même si le squat est subordonné à la ville en ce que celle-ci est potentiellement pourvoyeuse d'emplois, de services publics, de commerce etc. par la précarité des emplois qu'elle offre et le mode de vie et les codes qui s'y déploient et qui échappent aux jeunes kanak, la ville peut être perçue par ces derniers comme un lieu hostile. Face à la frustration relative à une difficile insertion institutionnelle et professionnelle, le squat devient un lieu de repliement, d'apaisement face à une ville dont les modes de vie ne sont pas adaptés et dont les codes ne sont pas maitrisés. La vie en squat en devient une façon de se faire une place en ville. Pour les jeunes rencontrés, le squat est leur territoire, mot qui au-delà de la localisation géographique renvoie à l'idée d'un espace identitaire, un lieu d'appropriation, investi de rapports sociaux et de liens ${ }^{89}$.

Pourtant la population nouméenne (celle non océanienne en particulier) porte très souvent un jugement dépréciatif sur ces squats et leurs habitants. Ils sont perçus d'une part comme portant atteinte au principe de propriété privée et d'autre part comme entachant la ville blanche, Nouméa (Dussy, 2005). Ainsi ses habitants sont considérés comme des citadins à part, "des étrangers à la ville», des gens de seconde zone, qui n'ont pas leur place dans l'espace urbain.

Dès lors on peut s'interroger sur la façon l'appartenance à ces zones d'habitation peut constituer un handicap supplémentaire pour l'insertion sociale d'une part et d'autre part la santé des individus en raison de la situation sociale précaire de ses habitants ou encore d'effet de désignation stigmatisant (Chauvin et Parizot (2005)).

Dans le cadre de notre étude, les squats de Magenta Soleil et de Sakamoto, ainsi que la maison de quartier proche du squat de Magenta Soleil, situés à Nouméa intra-muros, sont les lieux des rencontres effectuées. Les maisons de quartier sont des structures de proximité dédiées à l'animation de la vie du quartier, par des activités sociales, culturelles et ludiques. Plus ou moins actives en fonction des zones, elles peuvent être le lieu de rendez-vous de jeunes de quartier scolarisés ou non. Pour des raisons de discrétion et de confort, quelques jeunes ont préféré que les entretiens se déroulent dans ce lieu attenant au squat de Magenta soleil.

\section{Les structures médicales : Le Centre de Conseil Familial et l'ESPAS CMP}

21 Les professionnels de santé, plus précisément des médecins, ont été rencontrés en deux endroits: le centre de conseil familial (CCF) et le centre médical polyvalent (ESPAS CMP) lieux qui accueillent une importante population océanienne soit pour des dépistages anonymes et gratuits des infections sexuellement transmissibles, des conseils et une écoute sur des questions relatives aux interruptions volontaires de grossesse, à la contraception, aux problèmes de couple etc. 

raisonnable des lieux de soins retenus. Cependant l'absence de véhicule peut rapidement poser problème dans un contexte où les températures peuvent être élevées et que le relief vallonné de Nouméa ne facilite pas la marche à pied.

Concernant les bus, les changements nécessaires pour se rendre d'un endroit à un autre peuvent se révéler difficiles notamment pour des personnes accompagnées de jeunes enfants. A noter, certains squats en périphérie ne sont pas du tout (ou très mal) desservis en transport public.

Localisation des lieux de l'étude

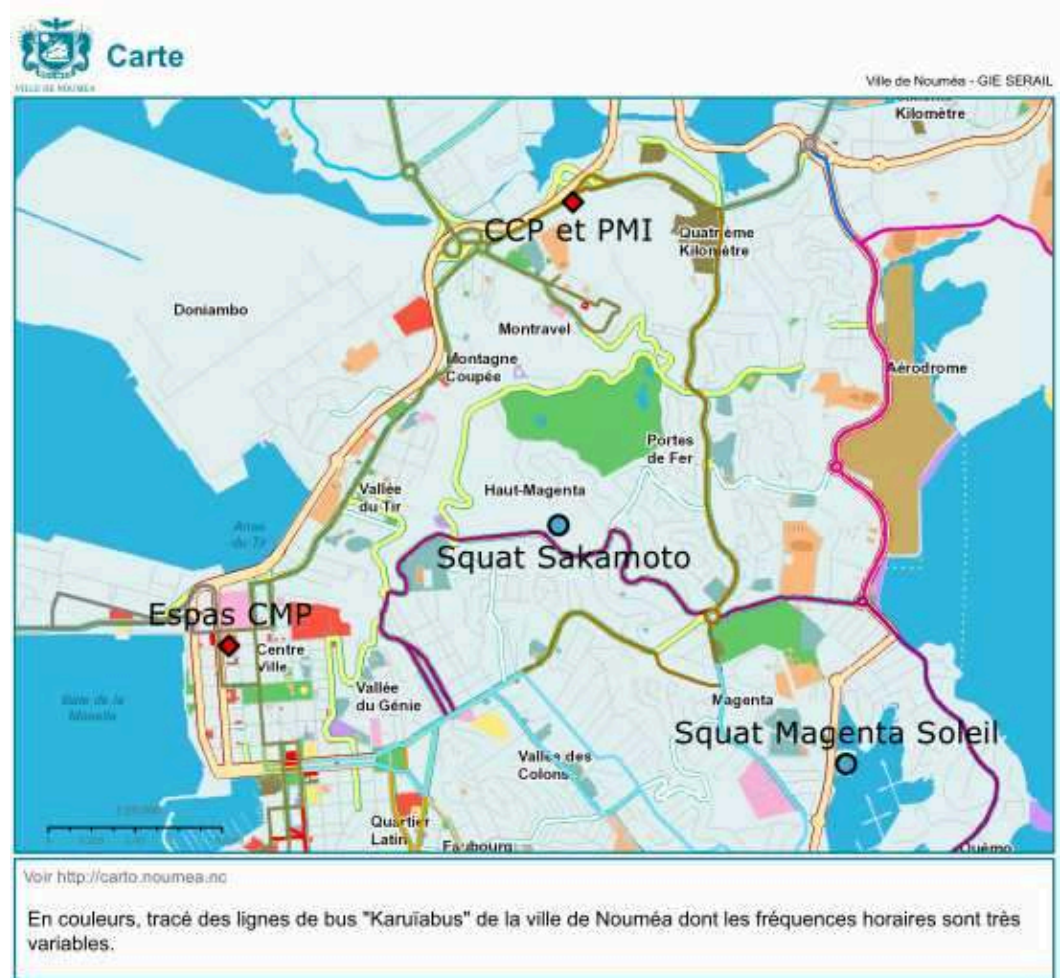

\section{Méthodologie et population de l'étude}

Une trentaine de jeunes kanak, se déclarant sexuellement actifs, a été rencontrée. Le premier critère retenu est l'âge des participants. Les jeunes devaient avoir entre 18 et 25 ans. L'autre critère retenu est le fait pour le jeune d'être sorti tôt du système scolaire sans diplôme et de ne pas occuper un emploi stable (en formation ou en CDD). Tous les jeunes de l'étude sont sortis du système scolaire sans diplôme. Le niveau d'étude le plus élevé est celui de la classe de troisième. Parmi les 31 jeunes rencontrés en squat, 18 femmes et 13 hommes. L'âge moyen est de 21 ans. Parmi les femmes 14 déclarent être célibataires et 4 en couple. Parmi les hommes, 11 sont célibataires et 2 en couple. Aucun des jeunes rencontrés ne dispose d'un contrat à durée indéterminée, 12 ont des emplois précaires ou des contrats de courtes durées comme manœuvre, ouvriers, femmes de ménage. Les 13 autres personnes sont sans emploi ou à la recherche d'un emploi.

L'accès au terrain, début 2014 a été facilité par une étude sur la santé sexuelle commanditée en 2010 par l'agence sanitaire et sociale. Certains jeunes interrogés 
en 2010 ont été de nouveau recontactés en 2014 et ceux qui correspondaient encore aux critères d'inclusion de l'enquête ont été réinterrogés. Des entretiens semi-directifs approfondis en face à face ont été menés auprès de ces jeunes par le même chercheur de mars 2014 à juin 2014.

Ont été abordés des sujets relatifs à leur enfance, leurs parcours scolaires, leur situation actuelle, leurs relations avec leurs familles et avec leurs partenaires mais aussi des sujets relatifs à leurs expériences antérieures avec les structures de soins, à leurs connaissances et à leurs expériences en santé sexuelle.

Des groupes de discussions souvent spontanés se sont également tenus dans le but d'une part de susciter l'échange et d'autre part de faciliter l'expression de certains jeunes plus à l'aise dans le groupe que dans la relation individuelle avec le chercheur. Toutes les personnes interrogées ont été volontaires pour participer à l'étude.

Six professionnels de santé et un responsable de maison de quartier qui mènent tous un travail de proximité auprès de cette population, ont également été consultés. L'objectif de ces entretiens était d'avoir un échange au sujet de la perception que ces professionnels ont des jeunes et de leur intervention auprès d'eux. Au total, sept personnes ont été rencontrées sur leurs lieux de travail. Les multiples réunions auxquelles j'ai participé lors de mes activités de chercheur et de consultante ont permis une observation participante de l'approche de la question «jeunes" par les professionnels de santé.

Cette étude étant qualitative, nous devons en préciser deux limites principales. La première est qu'elle n'est pas représentative de la population de jeunes vivant en squat. Le nombre de personnes interrogées étant peu important, l'étude ne couvre pas l'ensemble des profils socio-économiques qui s'y présentent. De plus, le choix de ne prendre en compte que les jeunes non-insérés renforce la spécificité de la population étudiée. Cette limite ne permet pas d'extrapoler les résultats à l'ensemble de la population jeune vivant en squat, encore moins à l'ensemble des jeunes de la NouvelleCalédonie. Cependant cette étude rend compte de la façon dont une certaine catégorie de jeunes vulnérables par leurs conditions de vie, leur faible niveau d'instruction, leur profil social et économique et leurs difficultés d'intégration sociale et professionnelle, profils régulièrement rencontrés dans ces lieux, perçoivent les questions relatives à la santé sexuelle et se comportent sur le plan sexuel.

30 La seconde limite est relative à l'ethnie d'appartenance des jeunes. L'étude ne couvre pas la diversité ethnique des squats: d'autres ethnies, notamment océaniennes, y résident. L'étude a été concentrée sur les jeunes de l'ethnie kanak pour des questions spécifiques à la Nouvelle-Calédonie d'un point de vue historique, politique et culturel. Ce sont les peuples autochtones du territoire, ceux qui ont subi la colonisation et qui revendiquent une reconnaissance de leur identité et de leur culture. Ils représentent $40 \%$ de la population de la Nouvelle-Calédonie. La population de l'enquête dont nous présentons les résultats ci-dessous, présente pour une bonne part les caractéristiques socio-démographiques d'une population jugée vulnérable aux IST : jeune, kanak, avec un faible niveau d'études, et composée majoritairement de personnes célibataires. 


\section{Des liens sociaux fragilisés favorisent des comportements de santé à risque}

31 Comme précisé plus haut, les ruptures sociales et/ou institutionnelles augmentent les risques pour les individus d'être confrontés à des difficultés sociales économiques et de santé (Chauvin et Parizot, 2005). Serge Paugam (2005) rend compte dans sa théorie sur le lien social, des risques et des conséquences individuelles de la rupture des liens sociaux, notamment chez les plus défavorisés. Ceux-ci disposent de peu de ressources ou de capital pour tisser et entretenir des liens suffisamment nombreux capables de leur assurer une stabilité. Comme le souligne l'auteur, la distension des liens sociaux conduit à un enchaînement d'" adversités » et de ruptures sociales qui se renforcent mutuellement. La rupture des liens a ainsi des conséquences négatives sur la protection et la reconnaissance (par la société) des personnes. Ces ruptures cumulées entraînent une disqualification sociale des personnes et renforcent leur marginalité ce qui affecte les différents secteurs de leur vie, dont celui de la santé.

32 Sur le territoire, de nombreux rapports ${ }^{10}$ font état de la situation préoccupante des jeunes et de leur mal-être. Dans cette phase de construction et de passage à l'âge adulte, les liens sociaux jouent un rôle prépondérant. Le lien joue un rôle fondamental dans la société kanak. Il donne sens aux relations entre membres d'une même famille, entre plusieurs familles d'un même clan, entre clans d'un même groupe, entre les ancêtres et les vivants. Ces liens plus qu'un concept sont « un mode de vie » qui permet aux personnes de s'inscrire et de circuler dans des espaces différents (Grochain, 2010). Une étude réalisée sous l'autorité du Sénat coutumier ${ }^{11}$ de la Nouvelle-Calédonie révèle la perte de liens auxquels sont confrontés les jeunes kanak qui ont " le sentiment d'être de plus en plus abandonnés par la famille, la coutume, la société civile et les institutions » (Lagadec, 2009).

33 Paugam définit entre autres trois types de liens sociaux à travers lesquels nous pouvons analyser la situation des jeunes kanak non-insérés vivant en squat.

34 En premier lieu, les liens familiaux considérés par Paugam comme ayant une fonction socialisatrice. Ils contribuent à l'équilibre de l'individu dès sa naissance en lui assurant à la fois protection (soins physiques) et reconnaissance (sécurité affective). Les jeunes rencontrés sont souvent pris dans des schémas familiaux complexes. Issus de familles séparées, recomposées, monoparentales ${ }^{12}$, leur parcours affectif et émotionnel est fragilisé. Ils sont la plupart du temps livrés à eux-mêmes, face à des parents restés à la tribu ou à des parents dépassés car eux-mêmes non insérés, des parents débordés qui doivent faire face aux exigences de la société capitaliste que leur impose la vie à Nouméa. Le Sénat coutumier dans son rapport (Lagadec, 2009) souligne le manque d'encadrement et de contrôle social des jeunes kanak notamment en milieu urbain, où ils sont extraits de la société traditionnelle, de la coutume et de ses exigences et doivent faire face à un monde urbain avec des us et des codes qui leur sont pour la plupart étrangers. On pourrait évoquer pour ces jeunes une sorte de désaffiliation (Castel, 1994) entendue comme un basculement dans un mode d'existence différent de celui de leur origine, désaffiliation favorable à leur propulsion dans une marginalité voire l'exclusion.

35 En second lieu, " le lien de participation élective » est défini entre autres comme le lien tissé dans le cadre de l'amitié ou de la relation de couple. Le jeune âge, une situation 
sociale et économique instable compromettant la possibilité de relation de couple stable, les liens amicaux sont surinvestis et se resserreront en général autour de copains et d'amis souvent dans la même situation sociale et professionnelle avec son lot d'incertitudes et de quête de sens. L'exclusion et le désœuvrement aidant, la bande des copains devient le lieu de socialisation mais également le lieu d'apprentissage et d'initiation aux addictions (cannabis, alcool) et aux actes délinquants. Les jeunes interrogés avouent leur dépendance à ces substances qui leur permettent de «tenir le coup » mais aussi qui les plongent dans des situations où ils n'ont aucune maîtrise de leurs comportements de santé (par exemple port du préservatif), ni de leurs comportements de façon générale (violence, violence sexuelle etc.).

En troisième lieu, le « lien de participation organique » est caractérisé selon l'auteur par l'apprentissage et l'exercice d'une fonction déterminée dans l'organisation du travail. Ce lien se constitue dans le cadre de l'école et se prolonge dans le monde du travail. Par définition, pour ces jeunes non-insérés, il est difficile voire impossible de développer ce lien de participation organique. Des contrats précaires ou de courte durée jalonnent leur parcours professionnel, certains s'étant même résignés à l'inactivité.

L'épanouissement dans une activité productive, la reconnaissance matérielle et symbolique, l'assurance de garanties face à l'avenir (Paugam, 2005) qu'offre un emploi font défaut à l'ensemble de ces jeunes non-insérés. C'est une fragilité supplémentaire des liens qui les rend d'autant plus vulnérables et affectent d'autres secteurs de leur vie dont celui de la santé. La pauvreté de ces jeunes et l'incertitude qui entoure leur vie, les confrontent à des problématiques qu'ils jugent autrement plus prioritaires que ceux de santé.

Prenons le cas de Georges, un jeune de 22 ans rencontré en squat. A la suite de la séparation de ses parents, il est accueilli dans quatre unités familiales différentes entre l'âge de 7 ans et de 14 ans. Il vit des épisodes de violence et se refugiera dans la relation étroite avec sa bande de copains. Après trois redoublements, il quitte l'école à 14 ans. Sa bande de copains prend de plus en plus de place dans sa vie. Sous l'emprise de l'alcool et/ou du cannabis ils se " défoulent " de temps en temps en taguant sur les murs du centre-ville ou en saccageant la maison de quartier. Sa consommation de drogues pour " tenir le coup " prend de plus en plus de place dans sa vie. Quelques rixes (conduite sans permis et en état d'ébriété, vol de voiture, etc.) lui ont valu un séjour en prison, prison qu'il qualifie de " parc pour kanak ». Par l'un des " tontons » de la bande de copains, il obtient de temps en temps de petits contrats comme manutentionnaire, mais il soutient que " les blancs riches " qui " profitent " de ce pays ne veulent pas de lui. Pourtant il dit être « chez lui ». Il ne veut pas retourner en tribu car il trouve que «les vieux sont difficiles ", ne " comprennent pas » les jeunes et " qu'il n'y a rien à y faire ». Il se retrouve souvent sans le sou en poche et peine à s'acheter à manger régulièrement. Il n'a pas de copine régulière, car il dit ne pas pouvoir " s'occuper " d'une famille. Il avoue avoir régulièrement des rapports non protégés notamment lors de fêtes sous l'emprise de drogues. Il ne sait pas s'il a déjà eu une IST car il dit ne pas aimer " les docteurs qui le jugent " et " voient le mal partout ». Il dit qu'il peut trouver des préservatifs gratuits au centre de santé. Il l'a fait une fois mais ne veut plus y retourner car les soignants lui ont " fait un mauvais regard ». Parfois, il envoie donc " les petits " en prendre pour lui. Il avoue être certains jours " découragé par la vie » et dit vouloir « arrêter tout ça » et ne pas vivre " dans ce monde sale ». De toute façon il pense n'avoir « rien à perdre " puisqu'il n'a « rien ». 
39 La rupture ou la fragilité des liens sociaux (famille, tribu, monde professionnel, institutions sanitaires) caractérise la vie de Georges. Instabilité, précarité et chaos jalonnent son parcours. L'effritement des liens sociaux entraine dans son cas une instabilité qui renforce sa disqualification sociale et sa marginalisation. En se référant aux travaux de Robert Castel (1994), on peut avancer que Georges par la précarité de sa situation professionnelle et la fragilité de ses soutiens relationnels est en " zone de vulnérabilité » extrême. Dans son parcours, la fragilité des liens étant présente depuis son très jeune âge, on ne peut évoquer le " décrochage » (Castel 1994) ni dans le cadre des relations d'une vie sociale ou professionnelle car aucune " accroche » stable n'est repérée dans sa trajectoire. A ce stade de sa vie, le squat, par le lien amical qu'il permet avec sa bande de copains, est le seul lieu qui lui permettre de rompre avec son isolement social. A ce titre le squat est surinvesti car il est le seul lieu où il peut prétendre à une place et à une identité sociales.

40 Dans ses propos, le « rien à perdre » est sous-tendu entre autres par l'absence de biens matériels, d'avenir, mais également de liens sociaux, un " rien à perdre » qui autorise de vivre sans se soucier d'affecter sa vie, car celle-ci l'est déjà largement. La prise de risque fait partie de son mode de vie. C'est même un engrenage de prises de risque général qui dépasse le seul cadre de la santé. Le risque dans le cadre de la santé (addictions, rapports non-protégés) s'inscrit dans une logique de prise de risques globale dans un contexte de distension de liens sociaux, d'avenir incertain et d'absence donné à son existence ${ }^{13}$. Dans ce contexte la santé et le comportement de santé ne sont pas la priorité de Georges dans l'échelle d'adversités à affronter.

\section{Sortie précoce du système et connaissances en santé sexuelle}

41 De nombreuses études ont établi une corrélation entre le niveau d'étude et les comportements de santé, le premier influençant favorablement les seconds.

Interrogés sur leurs connaissances en santé sexuelle, l'enquête révèle que certaines notions ne sont pas comprises des jeunes. Par exemple, chez les personnes rencontrées dans les squats, le sigle IST est très peu connu. Une fois le sigle explicité, les personnes en comprennent le sens (le rapprochement fait avec les maladies sexuellement transmissibles ou MST) et sont capables d'en citer quelques-unes, souvent de vagues souvenirs de cours de collège ou de messages de prévention. Dans l'ensemble de la population de l'étude, " la chaude pisse " est la maladie qui est la plus régulièrement citée. Ce nom expressif a su retenir apparemment l'attention et marquer la mémoire des personnes. Les symptômes des IST sont peu connus des personnes rencontrées sauf lorsqu'elles en ont été atteintes. Ainsi la perte de poids ou d'appétit ou encore la perte des cheveux ont été évoquées comme symptômes. Toujours concernant les IST, les lieux de dépistage du sida sont repérés, alors que ceux du dépistage des IST ne sont pas connus. Les jeunes interrogés évoquent vaguement l'hôpital ou le centre de protection maternelle et infantile (PMI) comme lieux de dépistage. Les personnes rencontrées savent cependant que ces maladies se transmettent essentiellement lors des rapports sexuels. L'existence de traitements est très peu connue sauf des personnes qui en ont fait l'expérience. Le terme même de " dépistage " n'est pas connu. Lorsque cette question a été abordée, de nombreux jeunes, notamment ceux des squats ont répondu : " dépistage veut dire quoi? ». En effet, de façon plus générale, la compréhension des 
termes médicaux posent problème. Il en va de même pour certains termes comme IST, VIH, séropositif, contraception, qui ne semblent pas être toujours des mots familiers notamment chez les hommes ayant un faible niveau de scolarisation.

43 En ce qui concerne les modes de transmission, un déficit de connaissance et des idées erronées persistent. Par exemple le fait d'avoir un rapport sexuel sans éjaculation est perçu comme une non-prise de risque. Ici le retrait du partenaire éviterait le risque de grossesse mais également le risque de maladie. La perception du risque n'est donc pas toujours très juste. Les propos des jeunes mettent également en évidence le fait que le risque de contracter une maladie se noie dans le risque d'" attraper " une grossesse. L'apparence du partenaire est considérée comme un élément de mesure du risque. Le risque est perçu comme moindre voire inexistant quand le partenaire est jugé d'apparence saine ou qu'il appartient à l'entourage familier (" je le connais depuis longtemps»).

44 L'ensemble de la population d'étude considère le VIH comme une maladie éloignée des réalités et de leurs préoccupations, même si elle est crainte car perçue comme mortelle. Sa transmission par voie sexuelle est celle qui est la plus citée et la mieux connue. La transmission par le sang est très rarement citée. La transmission materno-foetale n'est jamais citée. Un ensemble d'idées erronées circulent sur les modes de transmission parmi lesquelles le fait de boire dans le même verre, " de coucher avec une fille pendant ses affaires (ses règles) ", la fréquentation des toilettes publiques, etc. Quasiment toutes les personnes rencontrées avouent n'avoir jamais croisé une personne séropositive dans leur parcours. La distinction entre la séropositivité et la maladie déclarée n'est pas connue des jeunes. Concernant son dépistage, la prise de sang est fréquemment citée par les personnes rencontrées. Cependant, le dépistage par l'analyse d'urines est certaines fois avancé. Les personnes rencontrées citent également de nombreux signes extérieurs par lesquels on reconnaîtrait une personne séropositive, notamment l'amaigrissement. L'existence des médicaments antirétroviraux contre le sida est très peu connue de la population vivant en squat. Egalement le temps de prises et les effets des médicaments sont méconnus des populations rencontrées. La médecine traditionnelle est fréquemment citée dans les squats comme pouvant proposer des traitements contre le sida. Enfin, le traitement d'urgence post-exposition est méconnu de toutes les personnes rencontrées.

45 Les professionnels soulignent une " intellectualisation » des connaissances, c'est-à-dire le fait que les messages peuvent selon eux être connus et restitués par les jeunes comme le serait "une leçon " ou " un cours ", sans que ceux-ci modifient le comportement des personnes. En considérant les résultats de l'étude, il semblerait qu'une part conséquente des connaissances de cette catégorie de jeunes soit limitée voire erronée dans le domaine de la santé et plus précisément de la santé sexuelle. Ce manque de connaissance et d'informations sur les modes de transmission, les symptômes et les traitements des IST, affecte les comportements de santé des jeunes. En particulier le fait que cette population ignore la transmission materno-fœtale ou considère un rapport sexuel sans éjaculation comme dépourvu de risque, peut porter atteinte aux comportements de prévention.

46 De nombreuses activités de sensibilisation sont menées régulièrement et organisées en milieu scolaire. Il est le lieu de déploiement de nombreuses campagnes de prévention. Mais qu'en est-il de la réflexion et des stratégies pour atteindre les jeunes situés hors du circuit scolaire, universitaire ou des milieux professionnels? Il est nécessaire de 
s'interroger sur les lieux où les " atteindre » mais aussi de tenir compte de leur mobilité (navette entre la brousse, les îles et Nouméa) tout en favorisant leur repérage des lieux d'information, de dépistage et de prise en charge des IST. D'après les résultats de l'étude, les jeunes non insérés n'ont vraisemblablement pas acquis suffisamment de connaissances en santé sexuelle à l'école et n'ont pas assez d'outils qui leur permettent de forger leurs connaissances dans ce domaine précis de la santé. Les politiques de prévention, qui considèrent également comme acquis la plupart des termes (" dépistage ", "séropositif», "IST») gagneraient ainsi à prendre en compte l'importance de la dimension pédagogique que devraient revêtir leurs messages.

La question des connaissances en ce qu'elles participent éventuellement à l'orientation des comportements de santé, au recours aux soins, semble pertinente à prendre en compte dans les facteurs explicatifs de ces comportements.

\section{La différence culturelle en question}

48 Comme en Guyane (Carde, 2013), en Nouvelle-Calédonie l'origine des individus ne s'exprime pas uniquement par les catégories « républicaines ", à savoir, la nationalité ou le pays de naissance. L'origine des personnes est prise en compte dans les recensements de la population ${ }^{14}$ et de façon plus informelle dans la nomination et la classification de "l'Autre ».

49 En effet, l'identification et la nomination selon l'ethnie se déploient dans le cadre des relations sociales quotidiennes. Des comportements sont également volontiers associés à cette identification ethnique. Cette différenciation ethnique qui dérive vers une différenciation des cultures et des comportements qui en résultent, se déploie également dans des cadres plus institutionnels. Marie Salaün s'est intéressée à l'échec scolaire et à son étiologie en Nouvelle-Calédonie (Salaün, 2005). Elle souligne " la tendance de long terme à absolutiser la différence sur une base "culturelle» ». Elle révèle le fait que les explications contemporaines de l'échec scolaire semblent incapables de dépasser la perspective culturaliste. Qu'en est-il de la relation de soin et de l'explication des comportements de santé ? Comment la perception de l'Autre et de sa culture opèret-elle dans la grille de lecture des professionnels de santé face aux jeunes kanak? Comment l'approche culturaliste prend-elle le pas sur une approche culturelle, au détriment de la prise en compte des déterminants sociaux, économiques (déscolarisation, bas niveau d'études, inactivité, précarité, fragilité des liens) d'une part et des expériences et aspirations individuelles d'autre part.

50 Dans le cadre de la relation de soins, la désignation ethnique s'accompagne d'une catégorisation de chaque ethnie en fonction de " caractéristiques " propres. Ces supposées caractéristiques sont issues à la fois d'expériences et/ou d'histoires individuelles ou collectives, vécues ou racontées, mais également de l'Histoire telle qu'elle nourrit la mémoire collective. La différence culturelle est alors invoquée par les professionnels de santé en vue d'expliquer certaines incompréhensions et certains malentendus entre soignants et soignés ou des comportements de santé (Fassin, 2001). Dans le cadre de la santé sexuelle par exemple, il est commun d'entendre de la part des professionnels de santé que la question de la sexualité est taboue dans la culture kanak, ce qui compromet d'entrée les perspectives d'échanges dans ce domaine. Or ce postulat pose trois problèmes. D'une part, appréhender la question sexuelle sous l'angle d'un tabou spécifique à la population mélanésienne c'est d'une part faire abstraction de 
l'histoire et de l'actualité de la "question sexuelle » dans le monde et dans les sociétés ${ }^{15}$ et d'autre part nier l'évolution que peut connaitre une société. Aussi cette idée, posée comme une évidence voire « La » règle, bloque en amont, d'emblée, toute possibilité de dialogue ou d'échanges entre les professionnels et les soignés. Pourtant malgré le fait que les jeunes mentionnent la difficulté d'aborder ces questions dans leurs familles pour des raisons de respect et d'éducation, ils précisent se sentir plus à l'aise pour en discuter avec des professionnels de la santé. Ceux-ci pourraient donc être ici des vecteurs de connaissance. Enfin, considérer la sexualité comme tabou dans la société kanak ne laisse aucune possibilité de démarche critique réflexive dans laquelle le professionnel de santé interroge sa propre perception de la sexualité.

51 Les professionnels de santé, pour la grande majorité métropolitains, sont pour la plupart très demandeurs, à leur arrivée, de formation spécifique à l'approche culturelle de la maladie. Ces formations dispensées sur le territoire ont pour objectif selon l'organisme de formation ${ }^{16} \mathrm{de}$ " permettre aux "nouveaux arrivants", confrontés très rapidement dans leurs pratiques à des incompréhensions " d'avoir " une ouverture encore plus grande» et d'aider à "une compréhension enrichie (...) de l'Autre permettant ainsi (aux professionnels) une pratique plus efficiente ». Pourtant cette approche qui partirait probablement d'une bonne intention, peut avoir comme effet pervers moins qu'une ouverture, un enfermement de l'Autre (le patient) perçu essentiellement dans sa différence, l'Autre qui ne peut échapper au déterminisme de sa culture. Pour peu que l'approche ne soit pas nuancée ou relativisée par les professionnels, la distance culturelle peut s'en trouver amplifiée et la prise en compte de l'expression des individualités et des processus d'individuation en cours notamment en milieu urbain, complètement niée ou occultée. Cette approche pourrait également amplifier la catégorisation des individus sur la base d'une supposée différence culturelle et affecter les discours et les perceptions. Dans ce schéma, chaque groupe ethnique aurait ainsi un comportement spécifique de santé. L'approche en devient culturaliste. Or, du constat de la différence comportementale à la pratique professionnelle adaptée à cette différence, il n'y a qu'un pas (Carde, 2013).

52 Prenons un exemple : la réticence ou la réserve exprimées par les jeunes femmes kanak envers les moyens ou les méthodes contraceptives sont souvent perçues par les professionnels de santé comme un blocage culturel ou un manque de confiance envers les moyens de contraception. Pourtant, dans le cadre de l'étude, les femmes rencontrées ont surtout évoqué le rejet de ces moyens, sur la base d'expériences négatives (les implants "font saigner " ou désorganisent le cycle du corps par exemple). Ces expériences négatives ${ }^{17}$ pourraient expliquer dans certains cas, la réticence ou le rejet d'une contraception pourtant largement prescrite par les professionnels de santé qui la jugent plus adaptée et « plus tranquille » que la pilule par exemple ${ }^{18}$. Dans ce cas de figure, le sentiment pour les jeunes femmes de ne pas être entendues peut altérer la relation de confiance entre soignés et soignants et entraver le recours aux soins.

53 Les aspects culturels relatifs aux comportements de santé, les cultures locales de santé existent dans toutes les sociétés. Il ne s'agit pas de les nier. Dans le cadre de l'étude, par exemple, une jeune femme de 24 ans rencontrée dans les squats, dépistée pour une IST soutenait le fait que sa maladie pouvait être relative à la mauvaise relation qui la liait à son père. Tout en prenant les médicaments prescrits par son médecin, cette personne se pliera aux rites de " pardon » prescrits par la coutume pour « rétablir l'ordre » dans 
ses relations avec son père. Cependant, une vigilance s'impose dans la mesure où l'approche culturelle peut dériver en une approche culturaliste qui a tendance à réduire l'Autre à ses particularités culturelles en niant ses besoins et ses aspirations universels. (Massé, 2002). Elle peut également réduire la prise en compte des facteurs sociaux et économiques qui interférent dans la relation de soin d'une part et dans l'accès et le recours aux soins d'autre part.

54 En effet, l'approche culturaliste favorise l'occultation ou la minimisation de déterminants socio-économiques de la santé. Par exemple, l'absence ou le retard aux rendez-vous est souvent attribué par les professionnels à une incompréhension culturelle d'un besoin de suivi régulier sans que ne soient pris en compte les difficultés de déplacement pour certaines personnes, notamment celles défavorisées qui vivent en zones excentrées et ne disposent pas de véhicule. Une jeune mère kanak vivant en squat, âgée de 21 ans s'exprime en ces termes: " quand je dois aller au rendez-vous à la PMI, c'est compliqué de marcher avec les petits jusqu'au bus, avec la poussette, attendre le bus dans la chaleur, (...) en plus je change en ville, j'ai deux bus à prendre, redescendre, remonter (...) j'arrive toujours en retard à mes rendez-vous et les médecins ne sont pas contents ». Autre exemple, la difficulté à joindre les patients sur leur téléphone est attribuée à une perception culturelle différente de la continuité du soin ou à un rapport culturellement différent à la temporalité. Or cette difficulté pourrait simplement témoigner d'une instabilité de la vie sociale pour ces personnes. Il en est de même pour l'absence de cartes d'assurance maladie perçue certaines fois par les professionnels de santé comme une difficulté culturelle à anticiper les évènements. Dans le cadre de l'étude, quelquesunes des personnes rencontrées témoignent du caractère épique que peuvent revêtir les démarches administratives: Une jeune de femme de 23 ans vivant en squat s'exprime en ces termes: « ce n'est jamais le bon papier ou pas le bon endroit (...) on finit par se décourager ". Ainsi certaines personnes décident de ne consulter qu'en cas de " problèmes sérieux ", le parcours au sein de l'administration sanitaire ou sociale étant perçu comme difficile. Dans les squats, les personnes rencontrées perçoivent leur santé comme moyenne et déclarent " traîner " certains problèmes de santé, surtout ceux nécessitant la consultation d'un spécialiste, en particulier lorsqu'il faut prévoir une avance d'argent pour consulter. Cela témoigne de leur précarité sociale.

55 La mobilisation de la différence culturelle comme la source des incompréhensions n'est pas le seul fait des professionnels de santé. Les jeunes interrogés soulignent le fossé culturel qu'il peut exister entre eux et les professionnels de la santé qui n'ont pas la même culture ${ }^{19}$. Certains faits relatifs à la "coutume ${ }^{20}$ " ou à la médecine traditionnelle peuvent être tus de peur de ne pas être compris ou d'être " grondés » par les professionnels. Certains jeunes par exemple perçoivent la médecine traditionnelle kanak comme pouvant être efficace pour certaines affections et en font usage. Cependant ils ne mentionneront pas cette croyance, ni ne partageront leurs expériences à ce sujet avec les médecins de peur d'être incompris ou discrédités par une médecine occidentale perçue comme porteuse de logiques autres.

56 La distance sociale entre le soignant et le soigné doit également être prise en compte. Les jeunes rencontrés dans l'étude sont issus de milieux sociaux défavorisés et ont quitté le système scolaire avant d'acquérir les bases élémentaires (lire, comprendre, parler le français). Le fossé social mais aussi générationnel peut altérer les bases d'une relation de compréhension mutuelle et d'échange. Ainsi quelques jeunes ont souligné le fait que les professionnels les " prennent de haut ${ }^{21}$ ", les jugent et ne les écoutent pas. 
De par leur position sociale et professionnelle, ces professionnels peuvent en effet être perçus comme posant un regard de supériorité ou d'autorité sur les jeunes. Ces derniers estiment avoir un langage trop familier (argot, fautes de français) qui ne se prête pas à leurs entretiens avec les professionnels. Ainsi, ils préfèrent dans ces situations, être le moins loquace possible, ce qui limite les possibilités d'échange entre les deux partis. Ces mêmes personnes perçoivent également le langage des professionnels comme technique et n'en perçoivent pas toujours le sens sans "oser» demander des précisions. Ce mutisme interprété par un trait culturel kanak (" ils ne s'expriment pas ») est ici avant tout la conséquence d'une distance sociale entre les deux parties, ce qui entraîne une gêne chez les jeunes. Quelques professionnels, notamment des éducateurs spécialisés, avouent que le manque d'écoute et de temps des professionnels de santé peuvent faire défaut et les faire " passer à côté » d'échanges avec les jeunes. Ceux-ci ont besoin de s'exprimer, de poser des questions, de raconter leurs difficultés et pourraient le faire si davantage de temps et d'espace leur était accordés.

57 On peut également noter le fait que certains professionnels pourtant en charge socialement de ces populations joignent au discours culturaliste un discours défaitiste selon lequel l'intervention auprès de la population principalement kanak en squat " ne sert à rien " ou " n'est pas productif ». Dans ces cas, ils se contenteraient de " faire leur travail » avec un optimisme très relatif voire inexistant quant à l'efficacité de leur intervention, ce que perçoivent bien les soignés. Ceux-ci ne chercheront alors pas à communiquer ni à établir une relation de confiance.

Enfin, on peut noter en confrontant ces discours que la conviction des uns de ne pas être compris des autres semble être la principale entrave à une collaboration réussie. Ainsi, autant du côté des professionnels que des patients, chacun regarde l'autre comme ayant un espace culturel singulier avec des références et des repères qui lui sont propres. Ce regard fait parfois obstacle à la communication et à la compréhension entre les deux parties et peut laisser place à une tension latente qui pourrait entrainer un non-recours et une perte d'efficacité de la prévention et de la prise en charge des personnes.

59 L'approche par la différenciation culturelle présente dans les rapports sociaux quotidiens n'échappe pas à la relation de soin en Nouvelle-Calédonie. Si elle se déploie sur la base d'une essentialisation de la culture et d'un renforcement de l'altérité de l'Autre au risque de minimiser les déterminants sociaux de la santé, elle ne peut qu'entraver une relation de soin qui gagnerait à être pacifiée pour une meilleure efficience de la prise en charge.

\section{Conclusion}

60 Ce texte s'était fixé comme objectif de rendre compte de la façon dont une population doublement en marge d'une part par son manque d'insertion scolaire et professionnelle et d'autre part par l'excentricité ${ }^{22}$ de son lieu d'habitation (squats) fait face aux questions relatives à sa santé sexuelle. Il s'agissait plus précisément de rendre compte de la façon dont le niveau de connaissance de ces jeunes, la fragilité de leurs liens sociaux, la différence culturelle et sociale convoquée dans le cadre de la relation interethnique avec les professionnels de soin pouvaient impacter leur représentations et leurs comportements de santé. Une des limites de ce texte est de n'aborder que quelques aspects des déterminants sociaux de la santé. D'autres déterminants sociaux, 
politiques, historiques et ceux psychosociaux (l'estime de soi par exemple) sont bien évidemment à explorer et à mettre en corrélation dans une étude de dimension territoriale. Une autre limite propre à ce type de recherche réside dans la difficulté à établir le lien de cause à effet entre différences psychosociales ou socio-économiques et l'observation d'un différentiel sanitaire d'une part et d'autre part la quantification statistique de ces effets (Chauvin et Parizot 2005). Cette recherche présente également les limites d'une étude qualitative en ce que ces résultats ne sont pas généralisable à l'ensemble des jeunes kanak qui vivent en squats et encore moins à la population de jeunes en Nouvelle-Calédonie. Cependant l'intérêt de l'étude orientée vers la population de jeunes kanak non-insérés vivant en squat tient à plusieurs aspects.

61 En considérant la dimension politique et sanitaire, les jeunes sur le territoire font l'objet d'un ensemble de réflexions autour d'interventions à mener. Dans le domaine de la santé publique, la population jeune est l'objet de préoccupations multiples sur des sujets relatifs au taux de suicide, la consommation d'alcool, de cannabis, le tabac, les infections sexuellement transmissibles pour n'en citer que quelques-uns. La population jeune océanienne, notamment celle kanak semble payer un lourd tribut à l'ensemble de ces maux. L'étude met en lumière une catégorie de ces jeunes kanak, dans l'ombre parce que non-insérés c'est-à-dire qui par définition échappent aux espaces de classement habituel ${ }^{23}$ que sont l'école, les écoles de formation, l'université, le milieu professionnel, etc. Plus spécifiquement, l'étude révèle la façon dont la précarité de leur situation sociale (chômage, inactivité) et la fragilité, la rupture voire l'inexistence de liens sociaux et institutionnels affectent leurs ressources cognitives dans le domaine de la santé sexuelle, leurs comportements de santé, leur accès et leur recours aux soins. Les jeunes non scolarisés et non diplômés en ce qu'ils échappent en amont aux espaces et aux catégories de classement habituels, constituent une catégorie qui mérite toute l'attention des politiques et acteurs de prévention.

Dans un contexte d'inégalités et de disparités sociales avérées, les politiques publiques mises en œuvre quelles qu'elles soient, gagnent à prendre en compte et in fine à réduire les écarts entre les individus qu'ils soient relatifs au sexe, à l'ethnie, à l'âge ou à l'éducation. La complexité de la situation des jeunes kanak non-insérés et leur singularité dans le contexte calédonien posent la question de la production structurelle d'exclus dans une société. Les politiques à leur endroit qu'elles soient scolaires, éducatives, sanitaires, sociales, culturelles mériteraient la mise en œuvre d'actions particulières, en identifiant et en qualifiant en amont les ruptures voire l'inexistence de liens auxquels ils sont confrontés. La santé des jeunes est à relier à d'autres problématiques notamment celles relatives à leur insertion sociale, professionnelle et à leur pauvreté. Cette situation suggère une prise en charge globale des jeunes en précarité sociale et notamment des jeunes kanak grâce à une collaboration avec des institutions sociales, politiques, culturelles qui ont comme cible la population jeune. Cette collaboration permettrait une vision moins partielle de la problématique des jeunes et assurerait la mise en place de solutions adaptées. Sur le plan de la santé sexuelle, une politique intersectorielle associant les programmes de lutte contre les IST d'une part et ceux contre les addictions et la violence d'autre part, pourrait aider à mieux cibler et combiner les messages de prévention.

63 En considérant la dimension spatiale, l'étude apporte des éléments de lisibilité sur un espace très peu étudié, très méconnu et dont l'imaginaire est alimenté par des rumeurs et quelques épars faits divers. En effet, les squats sont des espaces certes subordonnés à 
la ville, mais qui dans leur implantation géographique tournent le dos à la ville (Dussy, 2005). Nichés dans le creux de vallée ou au cœur d'une faune luxuriante ou d'une mangrove proliférante, ces espaces sont "des invisibles à la ville» que les citadins devinent ou aperçoivent en sillonnant les routes en voiture. Cette invisibilité est à l'image de l'absence de politiques réels à l'endroit des jeunes non-insérés des squats, doublement « étrangers » ou " illégitimes » à la ville et qui tentent tant bien que mal de s'y faire une place à leur façon. Hors des institutions classiques (école, université, monde professionnel), leur zone d'habitation ne faisant pas l'objet de politique sanitaire précise et adapté, la question de l'information et de la promotion de la santé d'une part et des lieux où " toucher » les jeunes de ces zones d'habitation doivent être réfléchis et mieux ciblés des intervenants. La rue, par exemple, zone fortement investie socialement par ces jeunes, pourrait faire l'objet de réflexion comme lieu éventuel (entre autres) de déploiement de politiques éducatives et informatives. Ces messages gagneraient à être plus accessibles à ce public qui avoue ne pas toujours percevoir le sens des messages véhiculés. Des espaces où la parole est libérée, où questions et interrogations peuvent être abordées sans complexe ni tabou, sont également à concevoir pour ces jeunes qui ont souvent le sentiment d'être tiraillés entre le mode de vie et les représentations traditionnels et ceux modernes. Il convient surement d'avoir une lecture compréhensive des obstacles que rencontrent ces jeunes mais également de pouvoir repérer et rendre compte des ressources et des supports mobilisables par cette population pour faire face à ses difficultés.

Cette population de jeunes étant hors des espaces scolaire et professionnel, les lieux de soins peuvent être des lieux propices de leur sensibilisation et de leur information. Cependant l'étude attire l'attention sur la façon dont cet espace qui est le lieu de soins, est aussi un espace de déploiement d'une interrelation où l'ethnie et la culture de l'Autre occupe une place centrale dans un contexte de négociation d'une transition vers l'indépendance. Elle révèle la complexité de la relation interethnique entre les jeunes autochtones et les professionnels de santé majoritairement métropolitains, les effets de la distance sociale, et les limites d'une approche par la différenciation culturelle voire le culturalisme dans la relation de soin. Les professionnels de santé qui côtoient la population jeune pourraient être sensibilisés à la santé sexuelle (approche éducative, pédagogique) afin de pouvoir aborder la question de la sexualité et du dépistage VIH/ IST. Cette sensibilisation pourrait favoriser une communication plus fluide avec la population jeune. Une sensibilisation ou formation aux déterminants sociaux de la santé pourrait par ailleurs limiter une approche hâtive et culturaliste qui ne prend pas en compte les conditions et situations sociales et économiques dans lesquelles évoluent les jeunes. Celle-ci devrait favoriser le dialogue plutôt que le jugement en renforçant l'aptitude des professionnels à intégrer les codes et normes de la population jeune. Elle devrait également aiguiser leurs capacités à mettre en question leurs propres représentations. Ces différentes actions permettraient de limiter la distance sociale entre soignants et soignés et favoriseraient une communication dans des conditions plus sereines entre les deux parties.

65 Les jeunes en difficulté sociale et/ou affective peuvent nouer des relations avec des leaders communautaires ou religieux, rares remparts à leur souffrance affective. Ces leaders de communautés (présidents de squats, animateurs, éducateurs spécialisés, chefs religieux...) qui jouissent d'une proximité avec la population jeune pourraient être associés à la réflexion sur les modes de prévention appropriés et efficaces. 
66

$$
\begin{aligned}
& \text { comme groupe social entièrement à part et à part entière semble être une condition } \\
& \text { nécessaire pour forger le destin commun si cher aux Calédoniens. }
\end{aligned}
$$

également des acteurs incontournables de l'avenir. Leur porter un intérêt particulier

\section{BIBLIOGRAPHIE}

Agence Sanitaire de la Nouvelle-Calédonie (2011), Baromètre Santé, Résultats préliminaires

Apikaoua R., Briseul J.P. (2014), Le prêtre et le juge, Editions le Corridor bleue, 256 p.

Canales Selgado, I. (2009), La cohabitation du squat et du logement social en Nouvelle-Calédonie. Le cas de Nouméa et de l'agglomération nouméennes. Mémoire de master DEVTAT

Carde E., (2013), « Les uns et les Autres en Guyane, le jeu de l'interethnique sur la scène de l'accès aux soins », in Anthropologie et Sociétés, Université Laval, 2013

Castel R, (1994), « la dynamique des processus de marginalisation : de la vulnérabilité à la désaffiliation » in Cahiers de recherche sociologique , Numéro 22, 1994, p. 11-27

Chauvin P., Parizot I., (2005), Vulnérabilités sociales, santé et recours aux soins dans les quartiers défavorisés franciliens / Une analyse de la cohorte Sirs, sous la direction de Pierre Chauvin (Inserm) et Isabelle Parizot (CNRS)

Corsenac P., Noël M., Rouchon B., Hoy D., Roth A., (2014), « Enquête IST 2012 en NouvelleCalédonie : prévalences et facteurs sociodémographiques associés aux infections à Neisseria gonorrheae, à Chlamydia trachomatis et à Treponema pallidum », Bull Epidémiol Hebd .; (8): 144-52.

Dussy D. (2005). S'approprier la ville, Nouméa à l'image de ses Squats. Nouméa. 255 p. Téléchargeable sur le site suivant : http://hal.inria.fr/docs/00/03/41/43/PDF/ dussySapproprierlaville.pdf

Fassin D., (2001), Critique de la santé publique, une approche anthropologique Editions Balland, $371 \mathrm{p}$.

Gay JC. (2012), Les inégalités socio-spatiales: état des lieux, in Peuple premier et cohésion sociale en Nouvelle-Calédonie - Identités et Rééquilibrages, Faberon J.-Y. et Mennesson Th. (dir), Aix-enProvence, Presses universitaires d'Aix-Marseille, p. 189-203

Grochain C., « les liens du sens » in MWA VEE, in revue culturelle kanak, éditions Agence de développement de la culture kanak (ACDK) téléchargeable sur le site suivant : http:// mediatheque.adck.nc/mediath/Mwavee/Mwa_Vee_66-67_Adck_Web.pdf

Hadj Laure et al., (2012) «Vingt ans de politiques de rééquilibrage en Nouvelle-Calédonie : Démocratisation de l'école mais persistance des inégalités ethniques ", in Formation emploi 4/(n ${ }^{\circ}$ 120), p. 101-125

INSEE, Institut national de la statistique et des études économiques (2007), Recensement de la population en Nouvelle-Calédonie en 2004. Téléchargeable sur le site suivant : http:// www.insee.fr/fr/publications-et-services/irweb.asp?id=rpnc04 
INSERM (2008), Situation sociale et comportements de santé des jeunes en Nouvelle-Calédonie, téléchargeable sur le site suivant : http://www.ass.nc/etudes-et-recherches/donneescaledoniennes

INSERM (2011), Santé sexuelle des jeunes en Nouvelle-Calédonie, Agence sanitaire et sociale de la Nouvelle-Calédonie, téléchargeable sur le site suivant : http://www.ass.nc/etudes-et-recherches/ donnees-caledoniennes

Lagadec G., (2009), Entre tradition et modernité, la place du jeune Kanak. "La place du jeune kanak dans la société contemporaine et les moyens de lutter contre la marginalisation d'une partie de la jeunesse", Avis du Sénat coutumier, sur saisine de l'Etat. Octobre 2009

Martin L.K., (2013), Espèces d'espace : espaces de vie de jeunes en Nouvelle-Calédonie, Mai 2013, http://www.djs.gouv.nc/portal/page/portal/djs/librairie/fichiers/26672256.PDF

Massé R., (2002), « la santé publique en France », in Sciences sociales et santé , Vol 20, Num 20-4, pp. 141-149

Nguyen E., Bouyssou A., Lassau F., Basselier B., Sednaoui P., Gallay A., (2011), « Progression importante des infections à gonocoques en France : données des réseaux Rénago et RésIST » au 31 décembre 2009. Bull Epidémiol Hebd. (26-27-28):301-4. Téléchargeable sur le site suivant : http:// opac.invs.sante.fr/index.php?lvl=notice_display\&id=9575

Observatoire des territoires, (2011), Part des jeunes non-insérés, ni en emploi, ni scolarisés. http://www.datar.gouv.fr/observatoire-des-territoires/en/part-des-jeunes-non-inseres-ni-enemploi-ni-scolarises

Passa J. (2010), « les liens du sens » in MWA VEE, revue culturelle kanak, éditions Agence de développement de la culture kanak (ACDK). Téléchargeable sur le site suivant : http:// mediatheque.adck.nc/mediath/Mwavee/Mwa_Vee_66-67_Adck_Web.pdf

Paugam S., Les formes élémentaires de la pauvreté, Paris, PUF, 2005.

Prévost J.B. (2012), L'emploi des jeunes, Avis du conseil économique, social et environnemental, in les éditions des journaux officiels, $154 \mathrm{p}$ Téléchargeable sur le site suivant : http:// www.lecese.fr/sites/default/files/pdf/Avis/2012/2012_16_emploi_jeunes.pdf

Quercy P. (2002), les squats dans la région île de France, Situation et propositions, 39 p, Conseil général des ponts et chaussées. Téléchargeable sur le site suivant : http://www.jeudi-noir.org/ wp-content/uploads/2011/03/rap_2002-0030-01.pdf

Ris C., « Les inégalités ethniques dans l'accès à l'emploi en Nouvelle Calédonie », in Economie et statistique , $\mathrm{n}^{\circ}$ 464-465-466, avril 2014

Rivoilan P. et Broustet D., (2011), Recensement de la population en Nouvelle-Calédonie en 2009, Institut de la statistique et des études économiques de Nouvelle-Calédonie. Téléchargeable sur le site suivant : http://www.insee.fr/fr/themes/document.asp?ref_id=ip1338

Salaün M., (2005), De la mentalité primitive au choc des cultures. L'échec scolaire kanak et son étiologie : état des lieux. Téléchargeable sur le site suivant : http://www.mondecommun.com/ uploads/PDF/TexteSalaun.pdf

Sévres F., (2009), Les squats du Grand Nouméa : un phénomène urbain original Téléchargeable sur le site suivant : http://villes.blog.lemonde.fr/2009/12/05/les-squats-du-grand-noumea-unphenomene-urbain-original/

Taylor Nelson Sofres. Enquête (2008), Recensement des squats pour le compte de la Province Sud.

Wilkinson R., L'égalité c'est la santé, Ed. Demopolis, 2010, 251 p. 


\section{NOTES}

1. les statistiques des CDAG, l'étude de prévalence des IST, les statistiques d'activité des infirmeries du Vice Rectorat, auxquelles pourraient s'ajouter les données témoignant de la fréquence des infections génitales hautes, des grossesses extra-utérines et des Interruptions volontaires de grossesses dans cette tranche d'âge et les entretiens avec les professionnels de santé (Données hospitalières et celles du Centre de Conseil Familial).

2. Infections à Neisseria gonorrheae, à Chlamydia trachomatis et à Treponema pallidum. L'infection à Neisseria gonorrheae appelée blennorragie ou communément chaude pisse a longtemps été l'une des plus fréquentes IST. Sans traitement, les risques d'évolution vers une stérilité irréversible sont très importants, chez les deux sexes. L'infection à Chlamydia trachomatis ou la Chlamydiose touche en particulier les jeunes âgés de moins de 25 ans et représente la première cause d'infection sexuellement transmissible (IST) bactérienne en France. Ses symptômes sont imperceptibles. L'étude de prévalence sur le territoire identifie une prévalence de cette infection 6 fois plus élevée qu'en métropole. L'infection à Treponema pallidum ou Syphilis se manifeste par un chancre initial et par des atteintes viscérales et nerveuses tardives, certaines manifestations survenant plusieurs années après la contamination. En 2000, une recrudescence de cette infection est annoncée en France.

3. Il n'existe pas en Nouvelle-Calédonie de statistiques officielles précisant la part de médecins venus de métropole. Cependant selon mes sources, au moins $80 \%$ des médecins viennent de métropole. Il est également noté un important turn-over de ces médecins, notamment ceux en contrat avec le $\mathrm{CHT}$ et les remplaçants.

4. En 2009, 40,3\% des habitants de Nouvelle-Calédonie déclarent appartenir à la communauté kanak (99100 personnes). La deuxième communauté la plus représentée est celle des Européens : 29,2\% des déclarations, soit 71700 personnes. Suivent les Wallisiens et Futuniens, avec 8,7\% (21 300 personnes). Regroupées, les autres communautés identifiées représentent $7,3 \%$ de la population totale : Tahitiens $(2,0 \%)$, Indonésiens $(1,6 \%)$, Vietnamiens $(1,0 \%)$, Ni-Vanuatu $(0,9 \%)$, autres Asiatiques $(0,8 \%)$ et autres (1,0\%).(ISEE 2009).

5. Européens : 49,9\%, Kanak : 22,5\%, Polynésiens : 12,1\%, Asiatique : 5,9\%, Autres : 9,6\%.(ISEE 2009)

6. Sur le territoire, en dehors de ceux qui vivent en milieu urbain, certains kanak vivent en tribu. Celle-ci regroupe en un lieu les membres d'un ou de plusieurs clans.

7. L'idée ici n'est pas de dire que le squat correspond au mode de vie océanien, même si c'est l'argument souvent mobilisé, peut-être en raison de l'espace qui offre une possibilité de vie extérieure, de pratiquer l'agriculture, d'accueillir la famille etc. Le père Roch Apikaoua (2014), figure locale kanak déplore cette assimilation des squats au mode de vie océanien. Il qualifie les squats d'« habitation faite de bouts de tôle, de bouts de planche, ou de bouts de plastique » et les compare au match box sud-africain, habitats des townships issus de la politique ségrégationniste de l'apartheid. Il semble plus juste de qualifier les squats, d'alternative ou de solution de repli face aux logements pas adaptés et aux loyers élevés.

8. Comme le précise Dussy dans son étude, très peu de nouméens connaissent la vie effective des squats et très peu n'y ont mis les pieds, même si de nombreuses idées reçues et de rumeurs (pour la plupart négatives) circulent. 
9. Dans l'espace urbain, la rue représente également pour les jeunes, un autre espace de vie, le lieu d'expression des émotions, un espace de visibilité, espace intermédiaire entre le lieu d'habitation et le reste du monde (Martin, 2013)

10. Le rapport du Haut-commissariat et du gouvernement (2009), l'enquête de l'INSERM sur la situation sociale des jeunes et leurs comportements de santé (2008), le rapport du Sénat coutumier sur les jeunes (2009), le rapport du Conseil économique et social du territoire(2012), l'étude de Catherine RIS (2014) font un état des lieux plus détaillé de la situation sociale et économique des jeunes sur le territoire

11. Le Sénat Coutumier est l'assemblée des différents conseils coutumiers du pays kanak, il est saisi des projets et propositions de loi du pays ou de délibération relatifs à l'identité kanak.

12. Les enfants non reconnus par leur père peinent d'autant plus à asseoir leur identité. Ils sont qualifiés d'enfants « en l'air » dans la mesure où dans la société Kanak le père est celui qui donne la place et qui ancre (la racine).

13. Selon l'étude de l'INSERM (2008), $12 \%$ de jeunes Calédoniens de 16 à 25 ans ont déjà fait une tentative de suicide. Les taux en métropole et en Nouvelle-Zélande sont respectivement de $6 \%$ et $3,2 \%$.

14. Des questions similaires avaient été posées jusqu'en 1996, mais non au recensement de 2004. En Nouvelle-Calédonie, les neuf recensements effectués sur le territoire depuis la seconde guerre mondiale (sauf celui de 2004) ont tous identifié la communauté d'appartenance. La CNIL a donné un avis favorable au recensement de 2009 pour la Nouvelle-Calédonie, qui contient une question relative à l'appartenance des sondés à une «communauté " et, pour certains d'entre eux à une "tribu». La distinction se justifie depuis les accords de Matignon-Oudinot (1988) et de Nouméa (1998), qui ont défini une politique de rééquilibrage en faveur de la communauté kanak longtemps discriminée.

15. Nous faisons référence au tabou autour de la question sexuelle en France jusque dans les années 68, et des débats actuels (ou l'absence de débat) que génèrent encore dans les sociétés y compris celles dites modernes, la question de l'homosexualité, la sexualité des jeunes, des personnes âgées, des handicapés etc.

16. Les mots en italique sont extraits du site de l'institut de formation à l'administration publique (IFAP) à la référence suivante: http://www.ifap.nc/ index.php?option=com_content\&view=article\&id=211:la-societe-kanak-approcheculturelle-de-la-maladie\&catid=13:actualites\&Itemid $=64$

17. Des études scientifiques notent parmi les effets secondaires de l'implant la perturbation du cycle menstruel et des saignements issus de l'utérus.

18. Le Baromètre santé réalisé en 2010 révèle que 31,9\% des femmes mélanésiennes disposent de l'implant comme contraception contre $1,7 \%$ des européennes. Son efficacité sur une période de 3 ans semble être perçue par les professionnels de santé comme une parade à «l'inadaptation de la pilule » (prise quotidienne, observance) pour les jeunes femmes et à « l'irrégularité » de leurs consultations.

19. Dans un contexte de revendication d'une identité culturelle bien définie, qui les distingueraient des autres cultures, les kanak eux-mêmes se revendiquent de ces «traits culturels» que peuvent brandir leurs interlocuteurs pour les disqualifier. Des expressions telles que « les blancs ont la montre et les kanak ont le temps » mobilisés par 
les kanak est un pied de nez à ce que les occidentaux dont les professionnels de santé nomment un " rapport culturel particulier au temps et à l'espace ».

20. Le mot coutume est usuellement employé en Nouvelle-Calédonie pour désigner de façon générale la culture kanak, ses modes d'organisation sociale, ses traditions, ses valeurs et pratiques sociales et culturelles.

21. Dans ce cadre, on peut faire l'hypothèse d'une interférence du rapport colons/ colonisés dans la relation de soin. D'ailleurs à ce sujet, une étude historique qui rendrait compte de la prise en charge sanitaire et médicale de la population autochtone pourrait être éclairante.

22. Ce terme est à comprendre ici dans son sens géographique, c'est-à-dire comme caractère de ce qui est loin du centre, en périphérie, mais aussi dans acception sociale qui fait référence au décalage ou à la rupture avec les normes habituelles.

23. Je fais référence aux espaces de socialisation, d'inclusion et d'intégration. Les lieux d'enfermement ou d'exclusion comme la prison ne sont pas pris en compte dans cette recherche même si de nombreux jeunes kanak ont dans leur vécu un parcours carcéral. Selon le rapport du Sénat Coutumier de 2009, 98\% des jeunes interpellés sont kanak Il semble important de trouver ou de créer des espaces où capter cette population de jeunes, en amont et en dehors des lieux d'enfermement, pour des politiques sociales et sanitaires, éducatives ou informatives.

\section{RÉSUMÉS}

En Nouvelle-Calédonie, une étude épidémiologique de prévalence des infections sexuellement transmissibles, réalisée en 2012, révèle que le jeune âge, un faible niveau d'étude, le fait d'être une femme, l'appartenance ethnique constituent des facteurs sociodémographiques associés à un risque plus élevé dans les comportements de santé. Suite à ces résultats, la présente étude qualitative, réalisée auprès de jeunes kanak non-insérés qui vivent en squat dans le GrandNouméa, se penche sur la façon dont, la rupture de leurs liens sociaux, leur faible niveau d'études apportent un éclairage sur leurs connaissances et leurs comportements en matière de santé sexuelle. Nous examinerons également comment ces différents aspects sociaux échappent à la grille de lecture des professionnels de santé au profit d'une approche culturaliste.

In 2012 an epidemiological research concerning Sexual Transmitted Infections (STI) conducted in New Caledonia reveals that predominantly women, young people, less-educated people and members of the Kanak community tend to have the highest incidence of these infections than other groups. As a follow-up to that research, the current study is interested in the way how low level of education, a break-up of their social links and inter-ethnic relations have a strong effect on young Kanak people sexual health behaviours. In this context, are met on one hand vulnerable young Kanak (young indigenous people without a school-leaving qualification and with a low level of education) living in Grand-Nouméa squats and on the other hand health and social professionals.

In this survey, the low level of education questions the quality of knowledge about STI in the population being studied. Indeed, numerous researches reveal that education is connected to 
health and use of care. As a place of acquisition of knowledge, the school system can play directly this formative role within the framework of health or at least give people tools allowing them to meet their needs in health. Concerning young people who drop out of school early, questioning their cognitive resources concerning health and sexual health more particularly could reveal possible gaps or misunderstandings.

Other aspects are the social exclusion and the break-ups of social and institutional links this population of young people are facing. Numerous studies, in particular those of Pierre Chauvin and Isabelle Parizot in sensitive urban areas (2005), demonstrated that the experience of some break-ups increases the statistical risk of being confronted with other difficulties socioeconomic difficulties but also care recourse, identity problems. The authors specify that, generally speaking, for individuals, difficulties met in social and sanitary plans are problematic as the traditional forms of sociability and solidarity weaken. When social links slacken, risk of being confronted with a chain of adversities and social breaks increases. As we shall see later in this text, a larger share of the young urban Kanak population is confronted with these breaks that could compromise among others health behaviours.

In a context where Kanak people are longing for sovereignty, recognition and valuation of their culture in New Caledonia, the study reports the way interethnic relationships take form in a context of ethnic diversity. Studies led overseas, in particular those of E. Carde in Guiana (2013), underline the representations of others in care relation and the differentiated practices which ensue from it. We shall thus report speeches of health professionals who have for the greater part European origin and come from metropolitan France. We will analyse, in this particular context, the way cultural differentiation operates in care relationships and can interfere in care recourse.

\section{INDEX}

Mots-clés : MST, comportements sexuels, jeunes, squats, contexte socio-environnemental, Kanaks

Index géographique : Nouméa, Nouvelle-Calédonie

\section{AUTEUR}

\section{FATOU LEÏTY MBODJ}

Chercheure associée, ESO-Angers, Université d'Angers 